\title{
Design and Performance Analysis of Underwater Acoustic Networks
}

\author{
Andrej Stefanov and Milica Stojanovic
}

\begin{abstract}
We analyze the performance of underwater acoustic ad-hoc networks in the presence of interference. We assume a uniform distribution of nodes over a finite area, and focus for simplicity on a two-dimensional network. The node-to-node channel is modeled using frequency dependent path loss and Ricean fading. We adopt a communication theoretic approach and study the sustainable number of hops through the network as an indicator of the network connectivity, as well as power and bandwidth requirements. The network operation is highly dependent on the node density with two distinct regions of limited performance: the coverage-limited region, where the number of nodes in the network is small, and the interference-limited region, where the number of nodes is large. We show that a desired level of connectivity can be achieved through a judicious selection of the operating frequency, power and bandwidth. Numerical examples illustrate the results of the analysis.

These results motivate us to propose a hierarchical underwater acoustic sensor network architecture in which the sensors and the collector stations operate in distinct layers. The hierarchical architecture is supported by the property of the acoustic underwater transmission medium that for each transmission distance there exists an operating frequency for which the narrowband signal-to-noise ratio is maximized. The sensors and the collector stations are consequently allocated different operating frequencies. We assume a uniform distribution of both sensors and collector stations over the finite area of the sensing field, and model the channel accordingly. The analysis is performed under the assumption that there is interference from other nodes within the same layer of the hierarchy. Numerical examples illustrate the network performance and demonstrate that the preferred operating frequencies ensure network operation without any cross-interference between the collector network and the sensor network.
\end{abstract}

Index Terms-Underwater acoustic networks, ad-hoc networks, connectivity, hierarchical sensor networks.

\section{INTRODUCTION}

$\mathbf{T}$ HE DESIGN and analysis of underwater wireless (acoustic) communications systems is receiving an increased interest by both researchers and practitioners in the area [1], [2], [3]. Initial research efforts have focused on underwater acoustic channel modeling, link capacity analysis and pointto-point communications, (see [4], [5] and references therein). Motivated by these theoretical models and driven by the maturation of underwater acoustic modem technology [6], the development of underwater acoustic networks is coming close to realization. In order to facilitate these developments,

Manuscript received 15 November 2010; revised 26 April 2011. Research funded in part by ONR grant N00014-09-1-0700 and NSF grant 0831728.

A. Stefanov is with the Information Technology department, IBU Skopje, Macedonia (e-mail: andrejstefanov@ieee.org).

M. Stojanovic is with the Electrical and Computer Engineering Department, Northeastern University, Boston, MA, USA (e-mail: millitsa@mit.edu).

Digital Object Identifier 10.1109/JSAC.2011.111211. a further study and an improved understanding of underwater networking principles is of paramount importance. The design of underwater networks is afflicted by the harshness of the acoustic propagation medium. The limited, distance-dependent bandwidth, the poor quality of the physical link, as well as the associated deployment difficulties, preclude direct application of existing land-based radio network solutions, necessitating novel approaches in the design of underwater acoustic networks.

The study of underwater acoustic networks encompasses two very different directions. The first direction follows the paradigm of cellular systems with distributed nodes communicating through a fixed infrastructure of base stations [7]. The base stations may either be mounted on surface bouys and connected by radio links or be bottom mounted with a cable-based infrastructure. The second direction focuses on decentralized ad-hoc networks where there is no established infrastructure, and the nodes communicate through multihop relaying. While ad-hoc networks operating in terrestrial radio environments have been studied extensively [8], [9], the analysis of ad-hoc network performance in an underwater acoustic environment remains a very challenging and complicated task. There has been recent progress on the design of multiple-access methods [10], [11], [12], medium access control protocols [13], [14], [15], [16], [17], [18] and routing schemes [19], [20], [21], [22], [23] for underwater networks. These are very important considerations in their own right; nonetheless, they focus on certain aspects of the network's performance, rather than the behavior of the network as a whole. Therefore, there is still a need for an approach that would provide a perspective on the overall performance of underwater acoustic ad-hoc networks.

In this paper, we focus on an underwater network of bottom mounted nodes, i.e., a two-dimensional network topology. In underwater acoustic communication systems, the power is subject to high attenuation that depends both on the distance and the frequency of the signal, while the bandwidth is severely limited. Hence, we assume multihop transmission based on nearest neighbor routing, as it offers more beneficial bandwidth and path loss conditions [4]. As the physical layer plays a fundamental role in the performance of underwater acoustic systems, a bottom-up approach for the study of underwater acoustic networks emerges quite naturally. The capabilities and limitations of the physical layer in fact impact certain design parameters and the performance of the overall network. Hence, we adopt a communication theoretic approach [24] and investigate the ad-hoc network performance in the presence of interference from other nodes in the network. The node-to-node channel is subject to frequency dependent 
path loss and is modeled as Ricean fading [25], [26]. We focus on the interdependence between the sustainable number of hops in the network, as an indicator of network connectivity; end-to-end frame error probability, power, and bandwidth allocation. Our study reveals that the network operation is highly dependent on the node density with two distinct regions of limited performance: the coverage-limited region, where the number of nodes in the network is small, and the interferencelimited region, where the number of nodes is large.

We also introduce a hierarchical sensor network architecture in which the sensors and collector stations operate in distinct layers. The architecture is motivated by the properties of acoustic propagation. Namely, it exploits the frequencydependent attenuation, which implies that for each transmission distance, there exists an operating frequency for which the narrow-band signal-to-noise ratio is maximized. Multihop communications are established in the context of a data gathering protocol which conveys the information from the sensors to the collectors. As the distance between the sensors is shorter than the distance between the collectors, the sensor-to-sensor transmissions are allocated a higher operating frequency than the collector-to-collector transmissions. The orthogonality in the frequency domain guarantees that the sensor-to-sensor transmissions will not create interference for the collector-tocollector transmissions and vice versa.

We emphasize that in addition to the choice of communication architecture, the actual network design will be largely driven by the trade-offs between node costs, endurance, and sensor performance. Potential applications could include target tracking by a sensor field, submarine detection, mine countermeasures, etc., as discussed, e.g., in [3] and references therein.

The paper is organized as follows. Section II describes the underwater acoustic propagation model. The analysis of underwater acoustic ad-hoc networks is discussed in Section III. Section IV introduces the hierarchical sensor network architecture. Numerical results are presented in Section V. Section VI concludes the paper.

\section{Underwater ACOUStic ATtenuAtion AND Noise}

Underwater acoustic communication channels are characterized by a path loss that depends not only on the distance between the transmitter and the receiver, as is the case in many other wireless channels, but also on the signal frequency. The absorption loss increases with frequency, as well as with distance and limits the practically usable bandwidth. In this Section, we briefly overview the salient characteristics of acoustic propagation. A reader familiar with these aspects may wish to skip to the next Section.

\section{A. Attenuation}

Attenuation, or path loss, that occurs in an underwater acoustic channel over a distance $d$ for a signal of frequency $f$, is given by

$$
A(d, f)=A_{0} d^{\kappa} a(f)^{d}
$$

where $A_{0}$ is a unit-normalizing constant that includes fixed losses, $a(f)$ is the absorption coefficient and $\kappa$ is the spreading factor. In the case of practical spreading $\kappa=1.5$. The

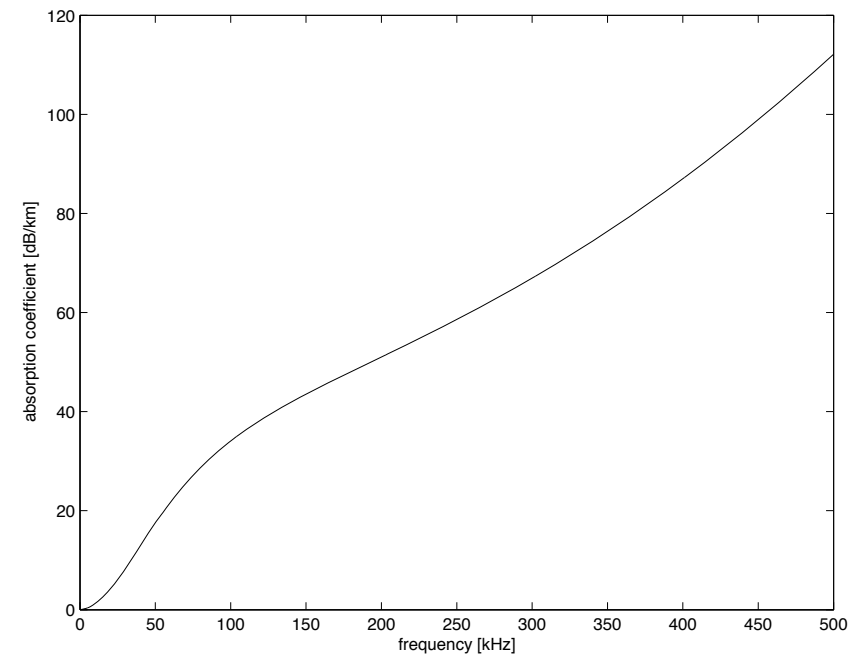

Fig. 1. The absorption coefficient $a(f)$ given in $\mathrm{dB} / \mathrm{km}$ for the signal frequency $f$ in $\mathrm{kHz}$.

absorption coefficient can be expressed empirically, using Thorp's formula which gives $a(f)$ in $\mathrm{dB} / \mathrm{km}$ for $f$ in $\mathrm{kHz}$ as [27]

$$
10 \log a(f)=\frac{0.11 f^{2}}{1+f^{2}}+\frac{44 f^{2}}{4100+f^{2}}+\frac{2.75 f^{2}}{10^{4}}+0.003 .
$$

This formula is generally valid for frequencies above a few hundred Hz. It is reproduced in Figure 1.

\section{B. Noise}

The ambient noise in the ocean can be modeled using four sources: turbulence, shipping, waves and thermal noise. Most of the ambient noise sources can be described by Gaussian statistics and a continuous power spectral density (p.s.d.). The following empirical formulae give the power spectral densities of the four noise components in $\mathrm{dB}$ re $\mu$ Pa per $\mathrm{Hz}$ as a function of frequency in $\mathrm{kHz}$ [27]

$$
\begin{aligned}
10 \log N_{\mathrm{t}}(f)= & 17-30 \log f, \\
10 \log N_{\mathrm{s}}(f)= & 40+20(s-0.5)+26 \log f \\
& -60 \log (f+0.03), \\
10 \log N_{\mathrm{w}}(f)= & 50+7.5 \sqrt{w}+20 \log f \\
& -40 \log (f+0.4), \\
10 \log N_{\mathrm{th}}(f)= & -15+20 \log f
\end{aligned}
$$

where $s$ is the shipping activity factor, $0 \leq s \leq 1$, and $w$ is the wind speed in $\mathrm{m} / \mathrm{s}$. The overall p.s.d. of the ambient noise is

$$
N(f)=N_{\mathrm{t}}(f)+N_{\mathrm{s}}(f)+N_{\mathrm{w}}(f)+N_{\mathrm{th}}(f) .
$$

\section{UNDERWATER ACOUSTIC AD-Hoc NETWORKS}

We analyze the performance of underwater acoustic adhoc networks, where the nodes communicate with each other without the presence of a central authority, under the constraint that a certain quality-of-service level, represented by a maximum allowed end-to-end frame error probability, needs to be maintained. In particular, we find that the network connectivity, in terms of the sustainable number of hops, 


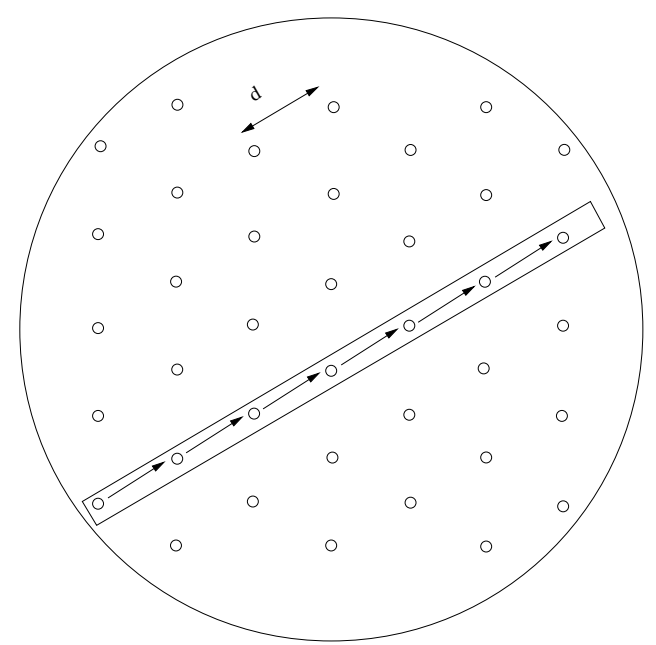

Fig. 2. A network with uniformly distributed nodes.

can be compromised by coverage, when the node density is too small, and by interference, when the node density is too high. Moreover, the range of node densities that support full connectivity depends on the choice of the transmit power and the operating frequency.

\section{A. Network Topology}

We focus on a two-dimensional network geometry, ${ }^{1}$ and consider a network of bottom mounted nodes. Let the area of the network be a circle of radius $r$. We assume a uniform distribution of nodes in the network as depicted in Figure 2. Given the number of nodes in the network, $N$, and the area of the network, $\mathcal{A}$, the density of the network is

$$
\rho=\frac{N}{\mathcal{A}} \text {. }
$$

Given the uniform node distribution, the distance between the nodes is

$$
d=\frac{c}{\sqrt{\rho}}
$$

where $c$ is a constant that depends on the node placement (grid pattern). Without the loss of generality, we let $c=1$.

We assume multihop transmission based on nearest neighbor routing. This is an energy saving strategy, hence it may be attractive for networks with battery-powered nodes. The analysis is performed under the assumption that the route discovery phase between the source and the destination has been successful. Further discussion regarding route discovery and the exchange of control messages may be found in [24] and references therein. As the longest multihop route in the network is along the diameter of the network, $D=2 \sqrt{\mathcal{A} / \pi}$, the maximum number of hops is

$$
n_{h}^{\max }=\frac{D}{d}=\frac{2}{\sqrt{\pi}} \sqrt{N} .
$$

Let the average number of hops for a multihop route be $\bar{n}_{h}$. As long as the probability distribution of the number of hops is symmetric, that is, as long as very long and very short routes

\footnotetext{
${ }^{1}$ Note that the approach can be readily extended to a three-dimensional topology.
}

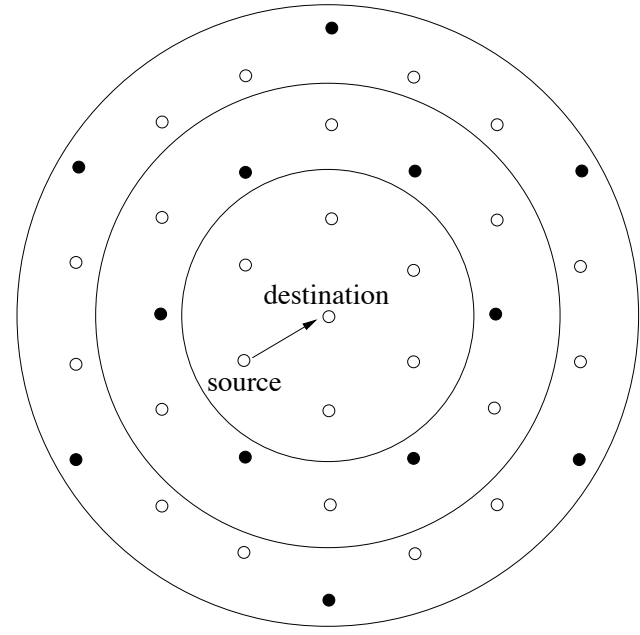

Fig. 3. Interference model. The black circles indicate nodes that are transmitting at the same time and on the same frequency as the source node (interfering nodes).

are much less likely than routes with an average number of hops, we have that [24]

$$
\bar{n}_{h}=\frac{n_{h}^{\max }}{2}=\sqrt{\frac{N}{\pi}} .
$$

\section{B. Interference Model}

In order to illustrate the effect of interference, we focus on a single transmission from a source node to a destination node, as depicted in Figure 3. We impose a protocol constraint: no nodes that are at the same distance from the destination node as the source node are allowed to transmit in the same time slot and in the same frequency band as the source during the source node's transmission.

The remaining nodes that may interfere with the source's transmission are organized in tiers. Assuming a hexagonal grid as an approximation of the network topology, there will be at most 12 interfering nodes in tier 1, 18 interfering nodes in tier 2, etc. A scenario where all the nodes in the network transmit at the same time is unrealistic, as some nodes will be receiving while others transmit. Figure 3 depicts a scenario where a half of the nodes in tier 1 and a third of the nodes in tier 2 transmit at the same time and in the same frequency band as the source, thus creating interference. Assuming that all the nodes transmit with some constant p.s.d. $S$, the combined interference from the nodes in the first and second tier is

$$
I(f) \approx \frac{c_{1} S}{A(2 d, f)}+\frac{c_{2} S}{A(3 d, f)}
$$

where $c_{1} \leq 12$ and $c_{2} \leq 18$ are constants indicating the number of interfering nodes in tiers 1 and 2 , respectively. In the example presented in Figure 3 , we have $c_{1}=c_{2}=6$. As there are multiple interfering nodes in the network, we assume that the interference is Gaussian with p.s.d. $I(f)$.

Using the attenuation $A(d, f)$, the noise p.s.d. $N(f)$ and the interference p.s.d. $I(f)$, we can evaluate the signal to interference plus noise ratio (SINR) observed over a distance $d$, as shown in Figure 4. We observe that there is a preferred operating frequency, $f_{o}(d)$, which depends on the distance, $d$. 


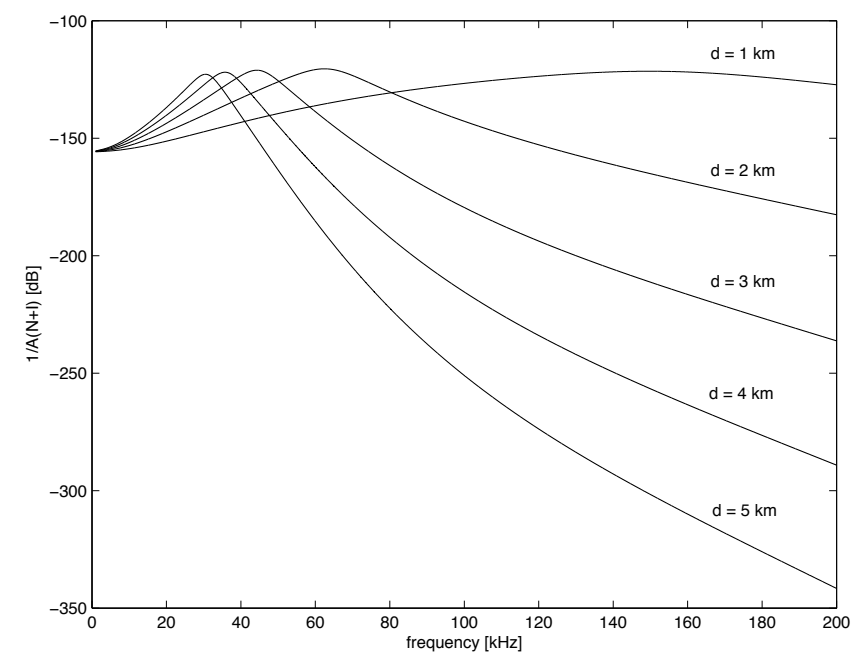

Fig. 4. $[A(N+I)]^{-1}$ for various distances $d$. The transmit p.s.d. is $S=150 \mathrm{~dB}$ re $\mu \mathrm{Pa}$ per Hz. The spreading factor is $\kappa=1.5$.

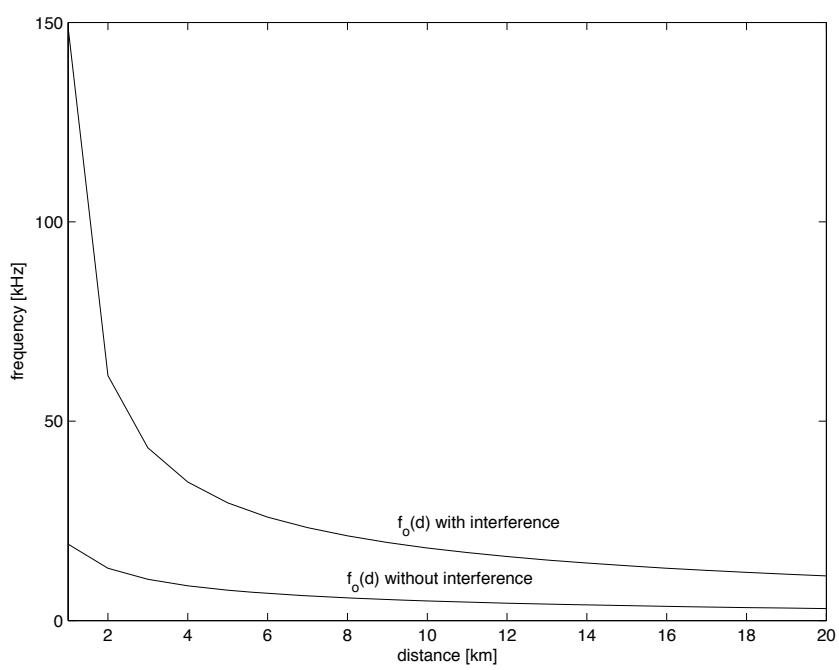

Fig. 5. Operating frequency $f_{o}(d)$ vs. distance for transmit p.s.d. of $S=150 \mathrm{~dB}$ re $\mu \mathrm{Pa}$ per $\mathrm{Hz}$. The spreading factor is $\kappa=1.5$.

The factor $[A(d, f)(N(f)+I(f))]^{-1}$ is maximized at this frequency. Figure 5 presents this preferred operating frequency as a function of the distance, given a transmit p.s.d. level ${ }^{2}$ of $S=150 \mathrm{~dB}$ re $\mu \mathrm{Pa}$ per $\mathrm{Hz}$ for $f$ in $\mathrm{kHz}$, while Figure 6 presents this preferred operating frequency as a function of the transmit p.s.d. for various distances.

\section{Multihop Transmission}

We assume a simple demodulate-and-forward relaying strategy. The end-to-end frame error probability (FEP) for a multihop route with $n_{h}$ hops is given by

$$
p_{\text {route }}=1-\left(1-p_{b}\right)^{L n_{h}}
$$

where $p_{b}$ denotes the bit error probability of a single node-tonode link, and $L$ denotes the frame size in bits.

${ }^{2}$ Note that an acoustic signal propagates as a pressure wave whose level is commonly measured in $\mathrm{dB}$ relative to $1 \mu \mathrm{Pa}$.

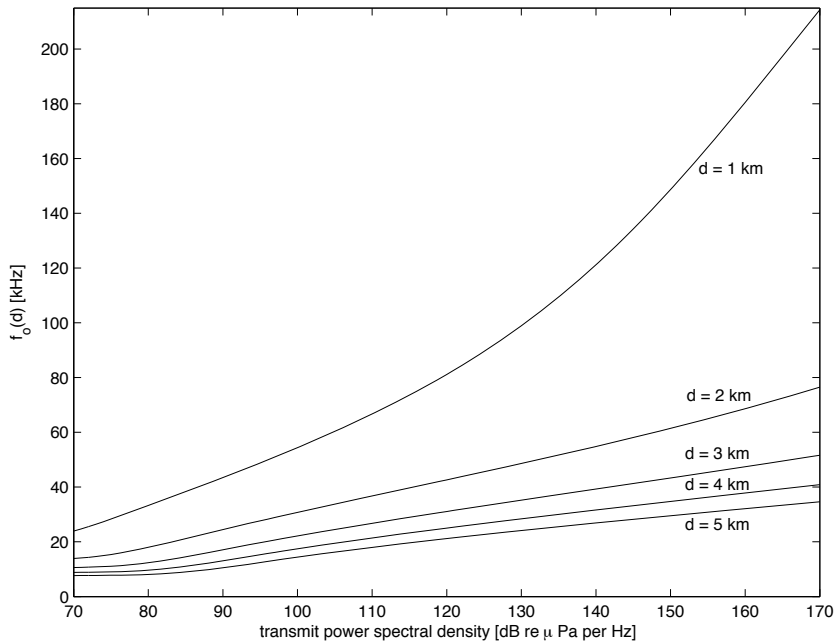

Fig. 6. Operating frequency $f_{o}(d)$ with interference vs. transmit p.s.d. for various distances. The spreading factor is $\kappa=1.5$.

We consider the network quality-of-service in terms of the maximum allowed end-to-end route FEP, i.e., we require that $p_{\text {route }} \leq p_{\text {route }}^{\max }$. Let the number of hops that can be sustained by the network, i.e., the number of hops that can satisfy the maximum end-to-end route FEP, be denoted by $n_{\text {sh }}$. From Eq. (10), it follows that $n_{\text {sh }}$ can be calculated as

$$
n_{\mathrm{sh}}=\frac{1}{L} \frac{\log \left(1-p_{\mathrm{route}}^{\max }\right)}{\log \left(1-p_{b}\right)} \approx \frac{1}{L} \frac{p_{\mathrm{route}}^{\max }}{p_{b}}
$$

While the analysis does not consider it explicitly, we note that in practice $n_{\mathrm{sh}}$ and $n_{h}^{\max }$ are chosen as nearest integers.

Without the loss of generality, we focus on uncoded BPSK transmission. Under the assumption of a Ricean fading model for the node-to-node channel [25], [26], and assuming that perfect channel state information is available at the receiver, the bit error probability ${ }^{3}$ can be upper bounded as [29]

$$
p_{b} \leq \frac{1+\mathcal{K}}{1+\mathcal{K}+\gamma(d, f)} \exp \left(-\frac{\mathcal{K} \gamma(d, f)}{1+\mathcal{K}+\gamma(d, f)}\right)
$$

where $\mathcal{K}$ denotes the Ricean fading factor and $\gamma$ denotes the SINR. We assume that the attenuation, noise and interference are constant over the operational bandwidth $B$, so that the SINR can be calculated at the operating frequency $f_{o}(d)$ as

$$
\gamma\left(d, f_{o}\right)=\frac{P}{A\left(d, f_{o}\right)\left(N\left(f_{o}\right)+I\left(f_{o}\right)\right) B} .
$$

Evaluating the SINR at $f_{o}(d)$ maximizes the SINR in Eq. (13), minimizes the node-to-node bit error probability $p_{b}$ in Eq. (12), and consequently maximizes the sustainable number of hops $n_{\text {sh }}$ in Eq. (11).

The frequency-nonselective assumption is a suitable approximation for systems with narrow bandwidth. It can also be extended to wideband multi-carrier communication systems, such as OFDM [30]. In that case, the operating frequency, $f_{o}(d)$, would describe the performance on one of the carriers. The performance on the other carriers would correspond

${ }^{3}$ Note that an illustration of the bit error probability for a Ricean fading channel with various fading factors $\mathcal{K}$ can be found in [29], Figure 13.7, p. 702. 


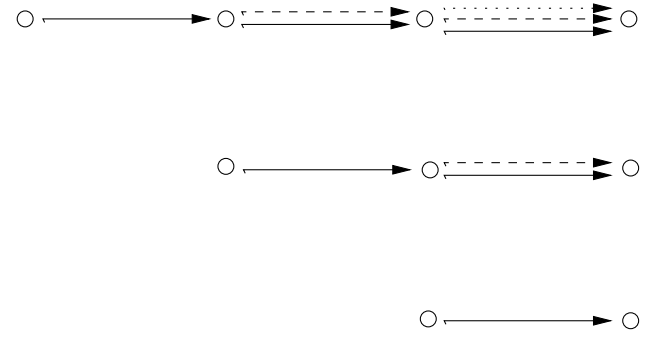

Fig. 7. Data gathering protocol.

to the frequency $f_{o}(d)$ shifted by multiples of subcarrier separation $\Delta f$.

\section{Hierarchical Sensor Network Architecture}

The findings of Section III, motivate a hierarchical underwater acoustic sensor network architecture in which the sensors and the collector stations operate in distinct layers. The bottom mounted sensors constitute the first layer in the architecture. The sensors are organized into disjoint cells, as illustrated in Figures 8 and 9. The sensors in each cell communicate their information utilizing multihop relaying to the collector station located at the center of the cell. The collector stations (collectors), which are also bottom mounted, form the second layer in the hierarchical architecture. The collectors similarly utilize multihop relaying to transmit their respective information to the central collector. As the distance between the sensors is shorter than the distance between the collectors, the sensor-to-sensor transmissions are allocated a higher operating frequency than the collector-to-collector transmissions.

\section{A. Data Gathering Protocol}

Both the sensors and the collectors utilize the same data gathering protocol. We consider two versions of the protocol and describe it in terms of sensor-to-sensor transmissions to the collector.

1) Protocol 1: The protocol is illustrated in Figure 7. The sensors closest to the collector transmit their information first in a single-hop transmission to the collector. The sensors that are two hops away transmit next through a two-hop route to the collector and so on. As they need to relay the data, the sensors closer to the collector can take advantage of the established route and transmit again. For example, Figure 7 shows sensors that are three hops away from the collector. They establish three-hop routes. The other sensors in the route, that are within two hops and a single hop from the collector, take advantage of the established route and send new information to the collector.

We note that spatial reuse of the bandwith is possible, so that there can exist multiple routes to the same collector. Of course, this leads to interference among transmissions utilizing the same time slot and the same bandwidth [28].

2) Protocol 2: The second data gathering protocol is a simplified version of the protocol described above. In this case, the sensors still utilize multihop transmissions; however, all sensors that are within the collector's cell transmit only once to the collector. In other words, sensors closer to the collector that are part of a multihop route for a sensor that is farther

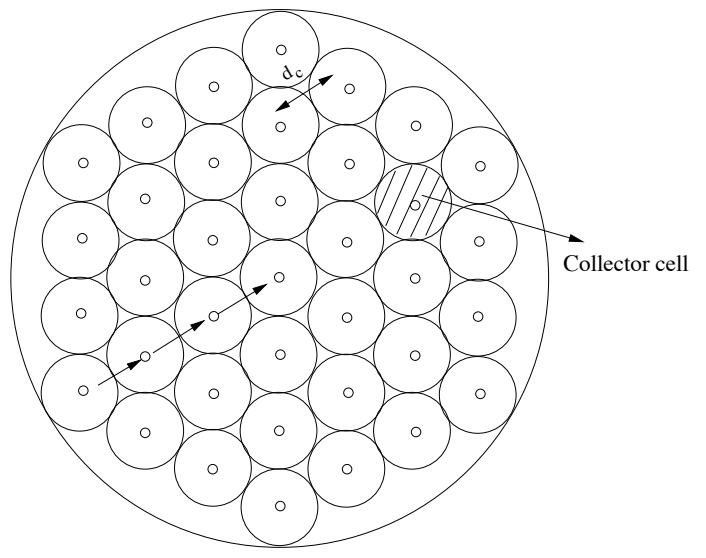

Fig. 8. Network of collector stations.

away from the collector only act as relays and do not transmit new information to the collector.

\section{B. Collector Network Topology}

We consider a network of bottom mounted collectors that is set-up in a manner analogous to the original network structure. The network of uniformly distributed collectors is shown in Figure 8. Given the number of collectors in the network, $K$, and the area of the network, $\mathcal{A}$, the density of the collectors in the network is $\rho_{c}=\frac{K}{\mathcal{A}}$. For a uniform collector distribution, the distance between the collectors is $d_{c}=\frac{c}{\sqrt{\rho_{c}}}$, where $c$ is a constant that depends on the grid pattern. As before, without the loss of generality, we let $c=1$, and we assume multihop transmission based on nearest neighbor routing. As the longest multihop route in the network is along the radius of the network, $r=\sqrt{\mathcal{A} / \pi}$, the maximum number of collector-tocollector hops is $n_{c}^{\max }=\sqrt{\frac{K}{\pi}}$. The average number of hops for a multihop route across the collectors is denoted by $\bar{n}_{c}$, and we have that $\bar{n}_{c} \lesssim \delta n_{c}^{\max }=\delta \sqrt{\frac{K}{\pi}}$, where $\delta$ is a constant that depends on the data gathering protocol. For example, in the case of protocol $1, \delta=\frac{1}{2}$; in the case of protocol $2, \delta=\frac{2}{3}$.

\section{Sensor Network Topology}

We consider a network of bottom mounted sensors. We assume a uniform distribution of $N$ sensors in the network. As there are $K$ collectors, there are $N_{c}=\frac{N}{K}$ sensors per collector, as depicted in Figure 9. The coverage area (cell) of each collector is $\mathcal{A}_{c}$. Hence, the density of the sensors is $\rho_{s}=\frac{N_{c}}{\mathcal{A}_{c}}=\frac{N}{\mathcal{A}}=\frac{1}{d_{s}^{2}}$. Following the steps of Section IV-B, we obtain the maximum and the average number of sensorto-sensor hops as $n_{s}^{\max }=\frac{1}{\sqrt{\pi}} \sqrt{\frac{N}{K}}$, and $\bar{n}_{s} \lesssim \delta n_{s}^{\max }$, respectively.

\section{Multihop Transmission}

We assume that both the sensor network and the collector network utilize uncoded BPSK transmission with a simple demodulate-and-forward strategy employed by the relays. Assuming the interference model presented in Section III-B and following the approach described in Section III-C, the number of hops that can be sustained by the network can be calculated 


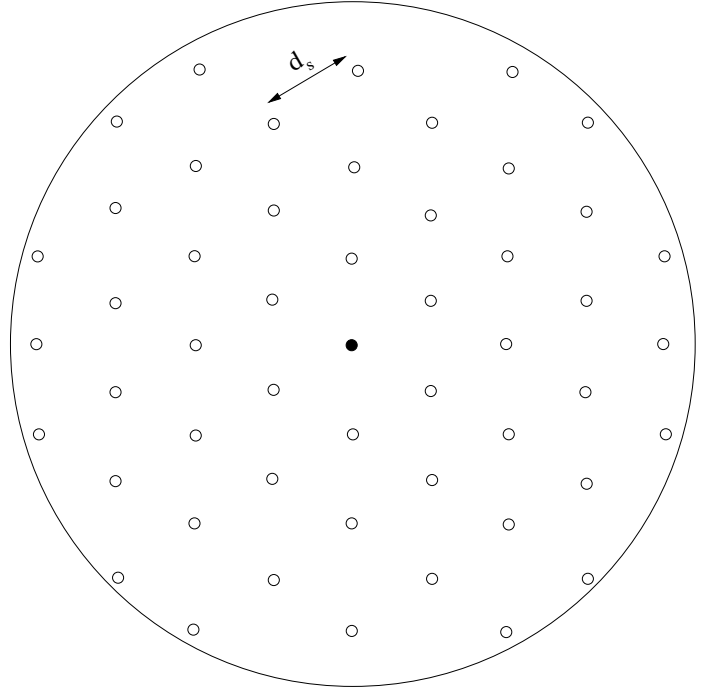

Fig. 9. A collector's cell contains $N_{c}=\frac{N}{K}$ sensors.

as, $n_{\mathrm{sh}} \approx(1 / L)\left(p_{\text {route }}^{\max } / p_{b}\right)$, where the bit error probability, $p_{b}$, is upper bounded by Eq. (12) and the SINR is given by Eq. (13). We note that while not considered explicitly in the analysis, in practice, $n_{\mathrm{sh}}, n_{c}^{\max }$, and $n_{s}^{\max }$ will be selected as nearest integers.

\section{NumericAl Results}

In this section, we present numerical examples that examine the relationships between the sustainable number of hops, the end-to-end FEP, the signal power, and the bandwidth. We assume a circular network of area $\mathcal{A}=1000 \mathrm{~km}^{2}$, the attenuation model given in Eq. (1), and we neglect any fixed losses. $^{4}$ We assume Ricean fading for each node-to-node channel [25], [26], [29]. The Ricean fading factor is taken to be $\mathcal{K}=10$. The spreading factor is $\kappa=1.5$, the shipping activity factor is $s=0.5$, and we assume calm conditions, that is, the wind speed is $w=0 \mathrm{~m} / \mathrm{s}$. The frame size is $L=100$ bits.

\section{A. Ad-Hoc Network}

Figure 10 presents the sustainable number of hops for a target (maximum allowed) end-to-end FEP of $p_{\text {route }}^{\max }=10^{-2}$, bandwidth $B=4 \mathrm{kHz}$ and transmit power $P=140 \mathrm{~dB}$ re $\mu \mathrm{Pa}$. The nodes are able to adjust their powers, so that the sustainable number of hops through the network never exceeds the maximum number of hops given in Eq. (7). The average number of hops given by Eq. (8) is also presented. We observe that when there are $N \lesssim 30$ nodes, the network cannot provide connectivity. This is due to the fact that with so few nodes in the network, the nodes are too far apart to guarantee the required end-to-end FEP for the available transmit power. Hence, the network is coveragelimited. Contrary to this situation, when the number of nodes in the network is $N \gtrsim 560$, we observe that the network can no longer sustain routes with the maximum number of

\footnotetext{
${ }^{4}$ Inclusion of additional frequency independent losses, and an adjustment of the background noise level to suit a particular environement and provide the necessary SINR margins, will scale the results in absolute value, but will not alter the general behavior.
}

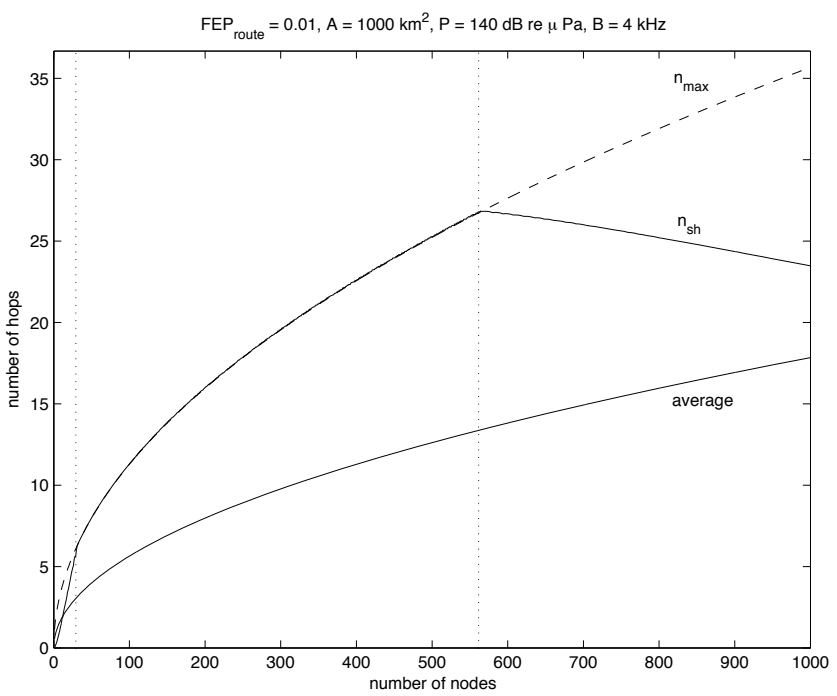

Fig. 10. Sustainable number of node-to-node hops for a uniform network with Ricean fading. The area is $\mathcal{A}=1000 \mathrm{~km}^{2}$, the bandwidth is $B=4 \mathrm{kHz}$, the transmit power is $P=140 \mathrm{~dB}$ re $\mu \mathrm{Pa}$.

hops. As we increase the number of nodes, while keeping the area of the network constant, the distance between the nodes decreases and the interference becomes stronger. Hence, the sustainable number of hops begins to decrease. We note, however, that even with $N=1000$ nodes in the network, the network can still maintain routes with an average number of hops. Nonetheless, the network is interference-limited. When the number of nodes in the network is between these values, $30 \lesssim N \lesssim 560$, the network can provide full connectivity and meet the target end-to-end FEP.

Figure 11 presents the sustainable number of hops for different values of the power. The required end-to-end FEP is $10^{-3}$. The bandwidth is $B=4 \mathrm{kHz}$. When the power is $P=168 \mathrm{~dB}$ re $\mu \mathrm{Pa}$ the network provides full connectivity for all values of $N<1000$. If the power is decreased to $P=164$ or $160 \mathrm{~dB}$ re $\mu \mathrm{Pa}$, the network becomes interference-limited when the number of nodes in the network is $N \gtrsim 700$ and $N \gtrsim 400$, respectively. The corresponding operating frequency and the minimum transmit power needed to achieve full connectivity, i.e., $n_{\mathrm{sh}}=n_{h}^{\max }$, are illustrated in Figure 12.

Figure 13 depicts the sustainable number of hops for different values of the bandwidth. The required end-to-end FEP is $10^{-3}$. The power is $P=164 \mathrm{~dB}$ re $\mu \mathrm{Pa}$. We observe that the network behavior changes as we vary the bandwidth. For example, when the bandwidth is $B=1 \mathrm{kHz}$ the network provides full connectivity for all values of $N<1000$. When the bandwidth is $B=2.5 \mathrm{kHz}$, the network becomes interference-limited when the number of nodes is $N \gtrsim 830$. When the bandwidth is increased to $B=4 \mathrm{kHz}$, the network becomes interference-limited when the number of nodes exceeds $N \gtrsim 700$. Note that this behavior is not inherent to the channel, but is rather caused by the fact that we have used the same signal power in all three cases. In other words, while the signal power remains the same, the noise power increases with the increased bandwidth causing an overall degradation in the system performance. 


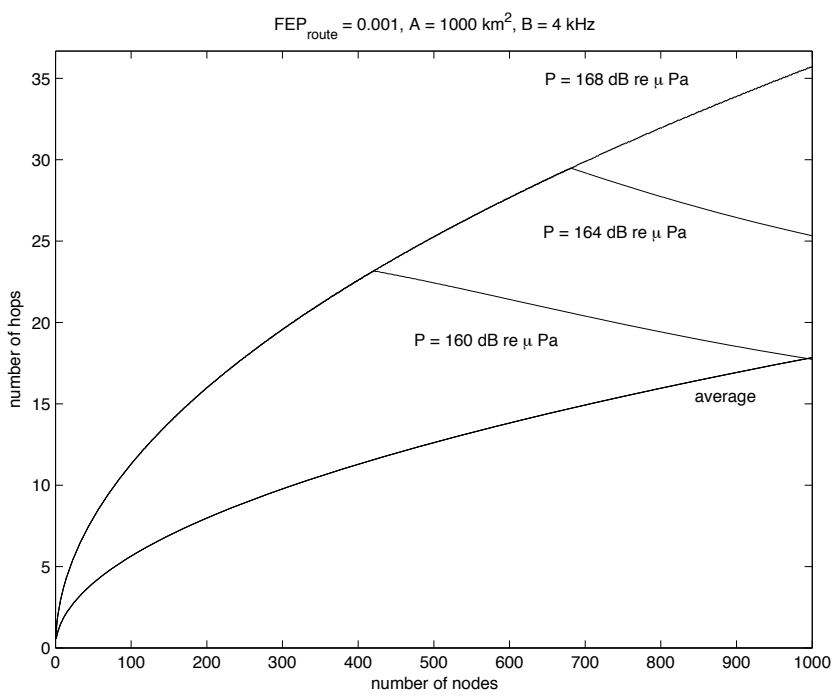

Fig. 11. Sustainable number of node-to-node hops for a uniform network with Ricean fading for various transmit powers. The area is $\mathcal{A}=1000 \mathrm{~km}^{2}$, the bandwidth is $B=4 \mathrm{kHz}$.
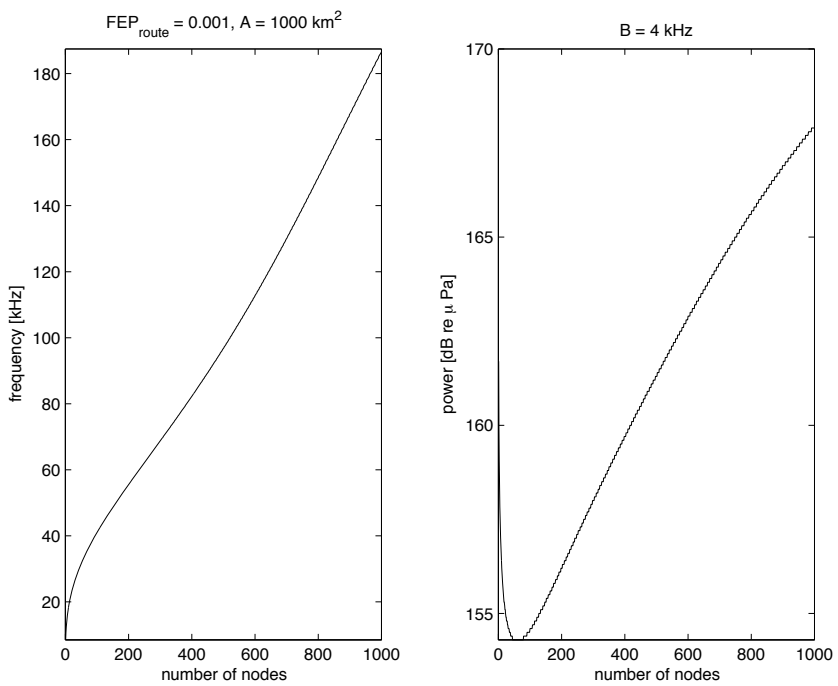

Fig. 12. The operating frequency and transmit power for a uniform network with Ricean fading. The area is $\mathcal{A}=1000 \mathrm{~km}^{2}$, the bandwidth is $B=4 \mathrm{kHz}$.

Figure 14 presents the sustainable number of hops for various values of the maximum allowed end-to-end FEP and transmit power. The bandwidth is $B=4 \mathrm{kHz}$. We observe that for a target end-to-end FEP of $10^{-3}$ and $P=164 \mathrm{~dB}$ re $\mu \mathrm{Pa}$, the network becomes interference-limited when the number of nodes is $N \gtrsim 700$. In this case the nodes have sufficient transmit power so that the network does not become coveragelimited when the number of nodes is small. When the target end-to-end FEP is $10^{-2}$ and $P=138 \mathrm{~dB}$ re $\mu \mathrm{Pa}$, the network can provide full connectivity when the number of nodes is between $70 \lesssim N \lesssim 300$. Above $N \gtrsim 300$ nodes, the network becomes interference-limited. In contrast, when the number of nodes in the network is less than $N \lesssim 70$ nodes, the network is coverage-limited.

The sensitivity of the sustainable number of hops to the carrier frequency is addressed in Figure 15. The required endto-end FEP is $10^{-3}$, the bandwidth is $B=4 \mathrm{kHz}$ and the

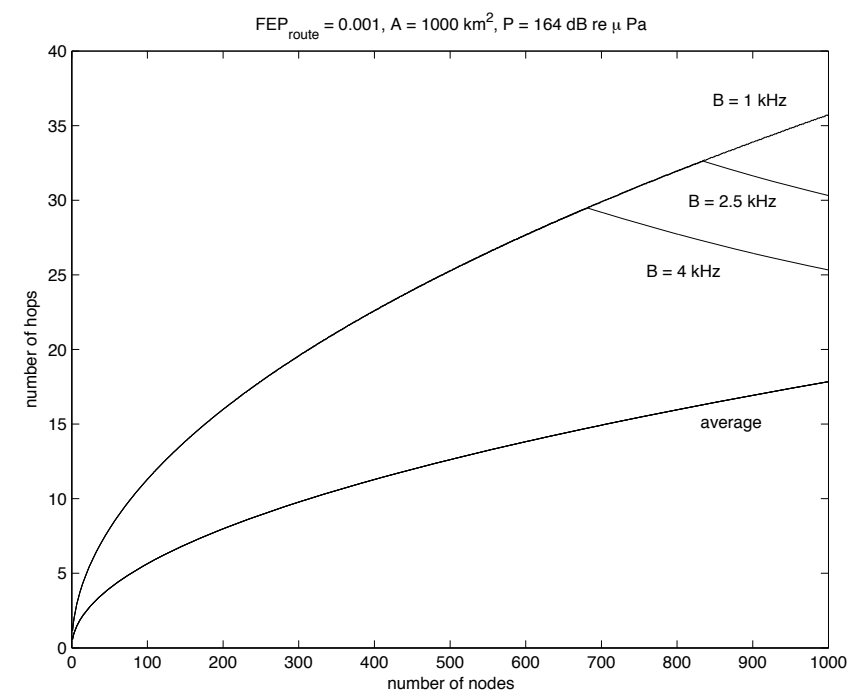

Fig. 13. Sustainable number of node-to-node hops for a uniform network with Ricean fading for various bandwidths. The area is $\mathcal{A}=1000 \mathrm{~km}^{2}$, the transmit power is $P=164 \mathrm{~dB}$ re $\mu \mathrm{Pa}$.

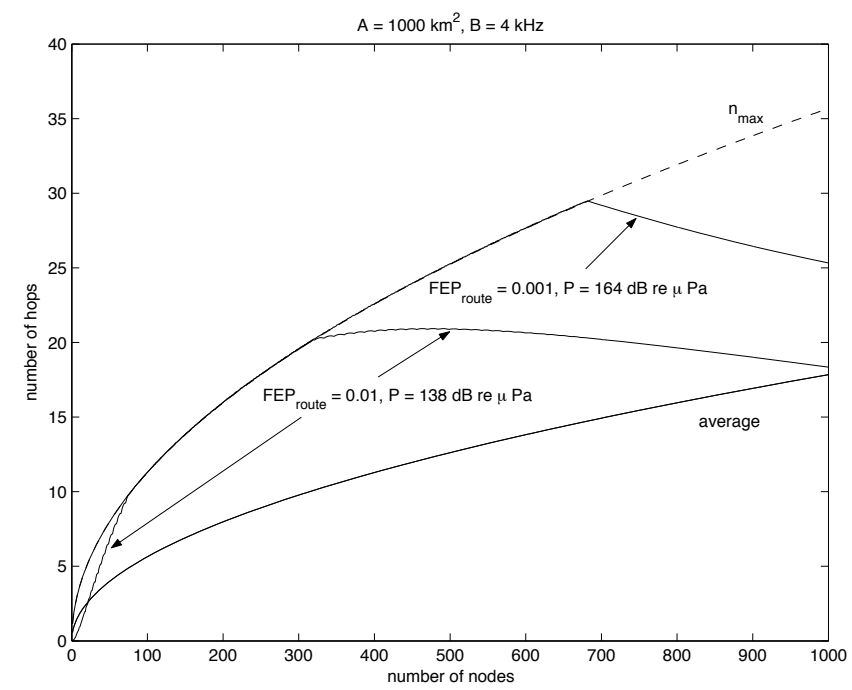

Fig. 14. Sustainable number of node-to-node hops for a uniform network with Ricean fading for various end-to-end frame error probabilities and transmit powers. The area is $\mathcal{A}=1000 \mathrm{~km}^{2}$.

transmit power is $P=164 \mathrm{~dB}$ re $\mu \mathrm{Pa}$. We observe that when $f_{o}$ is chosen as the operating frequency, the network becomes interference-limited when the number of nodes is $N \gtrsim 700$. At this frequency, the transmit power is sufficiently high that the network is not coverage-limited even for a small number of nodes. If the carrier frequency is $f_{o} \pm 5 \mathrm{kHz}$, the network becomes interference-limited when the number of nodes is $N \gtrsim 650$ and coverage-limited when the number of nodes is $N \lesssim 80$. When the carrier frequency is $f_{o} \pm 8 \mathrm{kHz}$, the changes in the operating regions of the network are more pronounced. We observe that when the number of nodes is $N \gtrsim 600$ nodes, the network becomes interference-limited. The network is also coverage-limited when the number of nodes in the network is $N \lesssim 250$. The network behaves in this way because a deviation from the preferred operating frequency $f_{o}$ causes the SINR to decrease, as depicted in 


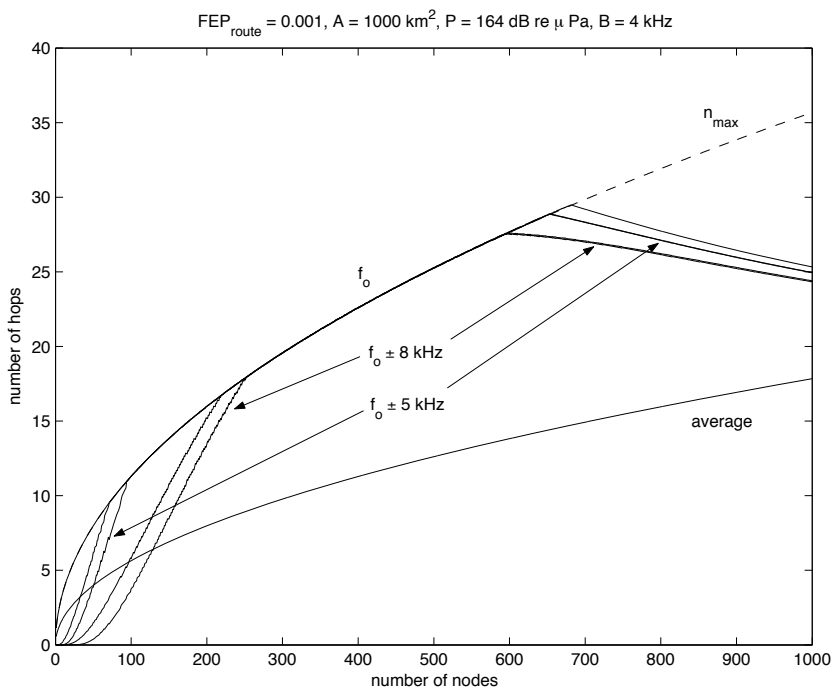

Fig. 15. Sustainable number of node-to-node hops for a uniform network with Ricean fading for various carrier frequencies: $f_{o}, f_{o} \pm 5 \mathrm{kHz}$ and $f_{o} \pm 8 \mathrm{kHz}$. The area is $\mathcal{A}=1000 \mathrm{~km}^{2}$, the bandwidth is $B=4 \mathrm{kHz}$, the transmit power is $P=164 \mathrm{~dB}$ re $\mu \mathrm{Pa}$.

Figure 4, and consequently, the sustainable number of hops decreases as well.

\section{B. Hierarchical Sensor Network}

We present numerical examples that examine the relationships between the sustainable number of hops, the end-toend FEP, the signal power, and the bandwidth. The target (maximum allowed) end-to-end FEP is $p_{\text {route }}^{\max }=10^{-3}$ for both the collector and the sensor networks. We assume that the hierarchical sensor network utilizes the data gathering protocol 1. Hence, the average number of collector-to-collector hops, $\bar{n}_{c}$, and sensor-to-sensor hops, $\bar{n}_{s}$, are calculated for $\delta=\frac{1}{2}$.

We present an example of a sensor network with 10000 sensors and 200 collectors deployed uniformly over a circular network of area $\mathcal{A}=10000 \mathrm{~km}^{2}$. Hence, there are $N_{c}=50$ sensors per collector. Each collector's coverage area is $\mathcal{A}_{c} \approx 50 \mathrm{~km}^{2}$. The performance of the collector network is presented in Figure 16. The collector's initial transmit power level is $P_{c}=152 \mathrm{~dB}$ re $\mu \mathrm{Pa}$ and the bandwidth is $B_{c}=4 \mathrm{kHz}$. The corresponding performance of each collector's cell sensor network is presented in Figure 17. The sensor's initial transmit power level is $P_{s}=148 \mathrm{~dB}$ re $\mu \mathrm{Pa}$ and the bandwidth is $B_{s}=4 \mathrm{kHz}$. We assume that the collectors and the sensors have the ability to adjust their power levels, so that the sustainable number of hops through the network never exceeds the maximum number of hops. The figures present the sustainable number of hops, the preferred operating frequency and the transmit power. Clearly, we require the sustainable number of hops to be equal to the maximum number of hops in order to guarantee full connectivity. As we observe from Figures 16 and 17, this is the case for both the collector network and the sensor network. We also observe that the operating frequency for the collector network is different from the operating frequency for the sensor network. For example, the operating frequency for the collector network when the number of collectors is $K=200$ is $f_{o}\left(d_{c}\right)=22.5 \mathrm{kHz}$. However, the operating frequency for the sensor network when the number of sensors per collector cell is $N_{c}=50$ is $f_{o}\left(d_{s}\right)=125 \mathrm{kHz}$. Hence, there is a sufficent frequency separation to ensure the operation of the hierarchical sensor network without any cross-interference between the collector network and the sensor network.

Figure 18 presents the sustainable number of sensor-tosensor hops in a collector cell for different values of the transmit power. ${ }^{5}$ The bandwidth is $B_{s}=4 \mathrm{kHz}$. The required end-to-end FEP is $10^{-3}$. We observe that the sustainable number of hops decreases as we decrease the sensors transmit power. For example, a transmit power of $P_{s}=148 \mathrm{~dB}$ re $\mu \mathrm{Pa}$ results in full connectivity in the collector cell: hence, data can be gathered from the entire area of the sensor field. However, if the transmit power is reduced to $P_{s}=140 \mathrm{~dB}$ re $\mu \mathrm{Pa}$, full connectivity cannot be achieved, as the network becomes interference-limited when the number of sensors in the collector cell is $N \gtrsim 32$. In other words, a reduction of the sensors power essentially leads to a reduction in the area of the sensing field of the respective collector cell.

\section{CONCLUSIONS}

We provided a communication theoretic analysis of underwater acoustic ad-hoc networks in the presence of interference. In particular, we studied the interdependence between the sustainable number of hops in the network, end-to-end FEP, power, and bandwidth allocation. We found that the region of the network where it provides full connectivity can be limited from below by coverage and from above by interference. When the number of nodes in the network is small, such that the available power is not sufficient to provide connectivity over a (large) area, the network is coveragelimited. As the number of nodes in the network increases while the network area remains constant, the distance between the nodes decreases, and the network becomes interferencelimited. Both the coverage-limited region and the interferencelimited region can be controled through a proper choice of the operating frequency and the transmit power. Specifically, when the operating frequency is chosen to maximize the SINR, the range of supported node densities (those for which full connectivity can be established) is widest. In contrast, if the operating frequency deviates from this value, or if the power is reduced from the minimum needed to achieve full connectivity, the range of supported densities narrows. We presented numerical examples that support these findings.

We also introduced a hierarchical sensor network architecture where the sensors and the collector stations operate in distinct layers. The hierarchical architecture was motivated by the fact that for each transmission distance there exists an operating frequency for which the SINR is maximized. We assumed a uniform distribution of both the sensors and the collectors over the finite area of the sensing field. The communication theoretic analysis supported by numerical examples showed that there is a sufficient frequency separation to ensure the operation of the hierarchical sensor network without

${ }^{5}$ Recall that these absolute values may scale depending upon the fixed losses and the noise level used for a particular system's link budget. 

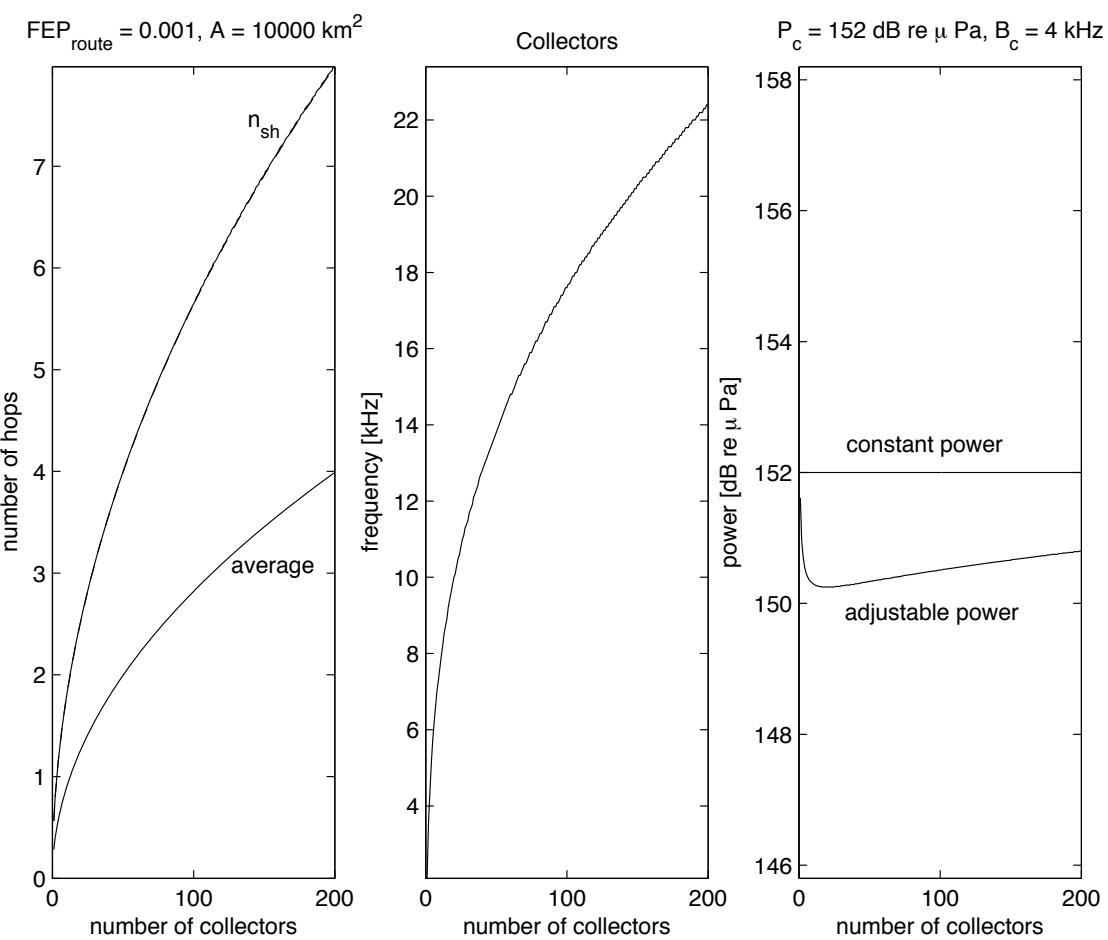

Fig. 16. Sustainable number of hops for a uniform network of collector stations with Ricean fading, operating frequency and transmit power. The bandwidth is $B_{c}=4 \mathrm{kHz}$.

any cross-interference between the collector network and the sensor network. This fact speaks in favor of a hierarchical architecture for the design of future underwater acoustic sensor networks.

\section{REFERENCES}

[1] Special Issue on Underwater Wireless Communications and Networks, IEEE J. Sel. Areas Commun., Dec. 2008.

[2] Special Issue on Underwater Networks, Ad Hoc Networks, Elsevier, Jun. 2009.

[3] R. Headrick and L. Freitag, "Growth of Underwater Communication Technology in the U.S. Navy," IEEE Commun. Mag., pp. 80-82, Jan. 2009.

[4] M. Stojanovic and J. Preisig, "Underwater Acoustic Communication Channels: Propagation Models and Statistical Characterization," IEEE Commun. Mag., pp. 84-89, Jan. 2009.

[5] M. Stojanovic, "On the Relationship Between Capacity and Distance in an Underwater Acoustic Channel," ACM SIGMOBILE Mobile Comp. Commun. Rev., vol. 11, No. 4, pp. 34-43, Oct. 2007.

[6] L. Freitag et al., "The WHOI Micro-Modem: An Acoustic Communications and Navigation System for Multiple Platforms," in Proc. MTS/IEEE Oceans Conference, pp. 1086-1092, Washington, D.C., Sep. 2005.

[7] M. Stojanovic, "Design and Capacity Analysis of Cellular-Type Underwater Acoustic Networks," IEEE J. Ocean. Eng., vol. 33, pp. 171-181, Apr. 2008.

[8] C. E. Perkins, "Ad Hoc Networking," Addison-Wesley, 2001.

[9] C.-K. Toh, "Ad Hoc Mobile Wireless Networks," Prentice-Hall, 2002.

[10] M. Stojanovic and L. Freitag, "Multichannel Detection for Wideband Underwater Acoustic CDMA Communications," IEEE J. Ocean. Eng., vol. 31, pp. 685-695, Jul. 2006.

[11] P. Casari, S. Marella, and M. Zorzi, "A Comparison of Multiple Access Techniques in Clustered Underwater Acoustic Networks," in Proc. IEEE OCEANS, Aberdeen, Scotland, 2007.

[12] L. Linton, P. Conder, and M. Faulkner, "Multiple-Access Communications for Underwater Acoustic Sensor Networks Using OFDM-IDMA," in Proc. MTS/IEEE OCEANS, Biloxi, MS, 2009.

[13] M. Molins and M. Stojanovic, "Slotted FAMA: a MAC protocol for underwater acoustic networks," in Proc. IEEE OCEANS Conference, Singapore, 2006.
[14] B. Peleato and M. Stojanovic, "Distance Aware Collision Avoidance Protocol for Ad-Hoc Underwater Acoustic Sensor Networks," IEEE Commun. Lett., vol. 11, pp. 1025-1027, Dec. 2007.

[15] M.-K. Park and V. Rodoplu, "UWAN-MAC: An Energy-Efficient MAC Protocol for Underwater Acoustic Wireless Sensor Networks," IEEE J. Ocean. Eng., vol. 32, pp. 710-720, Jul. 2007.

[16] A. A. Syed, W. Ye, and J. Heidemann, "Comparison and Evaluation of the T-Lohi MAC for Underwater Acoustic Sensor Networks," IEEE J. Sel. Areas Commun., vol. 26, pp. 1731-1743, Dec. 2008.

[17] H.-N. Ng, W.-S. Soh, M. Motani, "MACA-U: A Media Access Protocol for Underwater Acoustic Networks," in Proc. IEEE GLOBECOM, New Orleans, LA, 2008.

[18] X. Guo, M. Frater, and M. Ryan, "Design of a Propagation-DelayTolerant MAC Protocol for Underwater Acoustic Sensor Networks," IEEE J. Ocean. Eng., vol. 34, pp. 170-180, Apr. 2009.

[19] E. A. Carlson, P.-P. Beaujean, and E. An, "An Improved Location-Aware Routing Protocol for Mobile Underwater Acoustic Networks," in Proc. IEEE OCEANS Conference, Vancouver, Canada, 2007.

[20] N. Nicolaou, A. See, P. Xie, J.-H. Cui, and D. Maggiorini, 'Improving the Robustness of Location-Based Routing for Underwater Sensor Networks," in Proc. IEEE OCEANS Conference, Aberdeen, Scotland, 2007.

[21] M. Zorzi, P. Casari, N. Baldo, and A. F. Harris III, "Energy-Efficient Routing Schemes for Underwater Acoustic Networks," IEEE J. Sel. Areas Commun., vol. 26, pp. 1754-1766, Dec. 2008.

[22] D. Pompili, T. Melodia, and I. F. Akyldiz, "Distributed Routing Algorithms for Underwater Acoustic Sensor Networks," IEEE Trans. Wireless Commu., vol. 9, pp. 2934-2944, Sep. 2010.

[23] N. B. Qadri and G. A. Shah, "Performance Evaluation of Ad-Hoc Routing Protocols in Underwater Acoustic Sensor Networks," in Proc. Wireless and Optical Communications Conference (WOCC), Shanghai, China, 2010.

[24] O. Tonguz and G. Ferrari, "Ad Hoc Wireless Networks: A Communication-Theoretic Perspective," Wiley, 2006.

[25] P. Qarabaqi and M. Stojanovic, "Statistical Modeling of a Shallow Water Acoustic Communication Channel," in Proc. Underwater Acoustic Measurements Conference, Nafplion, Greece, Jun. 2009.

[26] A. Radosevic, J. Proakis, and M. Stojanovic, "Statistical Characterization and Capacity of Shallow Water Acoustic Channels," in Proc. IEEE OCEANS Conference, Bremen, Germany, May 2009.

[27] L. Berkhovskikh and Y. Lysanov, "Fundamentals of Ocean Acoustics," Springer, 1982. 

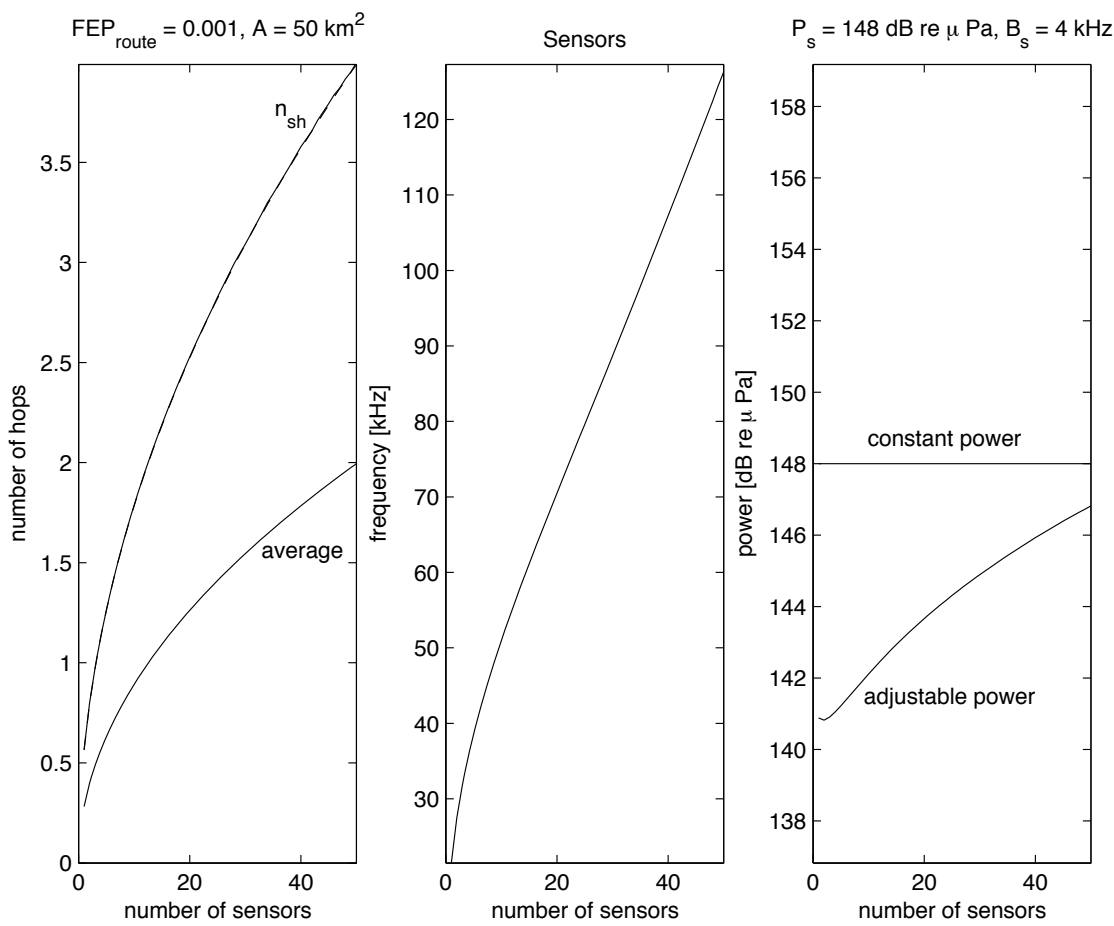

Fig. 17. Sustainable number of hops for a uniform distribution of sensors in a collector cell with Ricean fading, operating frequency and transmit power. The bandwidth is $B_{s}=4 \mathrm{kHz}$.

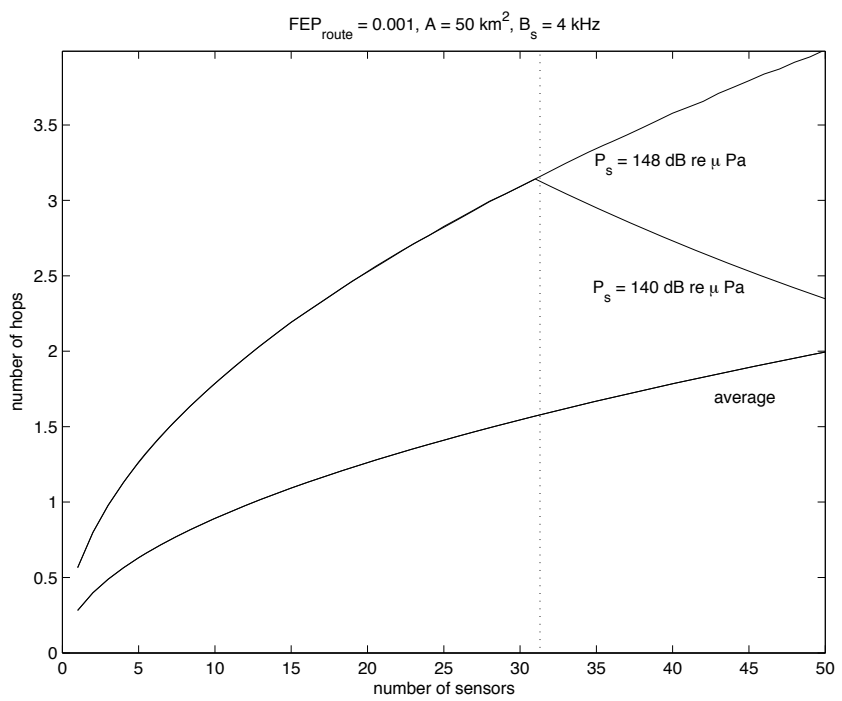

Fig. 18. Sustainable number of hops for a uniform distribution of sensors in a collector cell with Ricean fading for various sensors powers. The bandwidth is $B_{s}=4 \mathrm{kHz}$.

[28] W. Zhang, U. Mitra, and M. Stojanovic, ”Analysis of a Linear Multihop Underwater Acoustic Network," IEEE J. Ocean. Eng., vol. 35, pp. 961970, Oct. 2010

[29] S. Benedetto and E. Biglieri, "Principles of Digital Transmission with Wireless Applications," Kluwer/Plenum, 1999.

[30] L. Hanzo and T. Keller, "OFDM and MC-CDMA: A Primer," WileyIEEE Press, 2006.

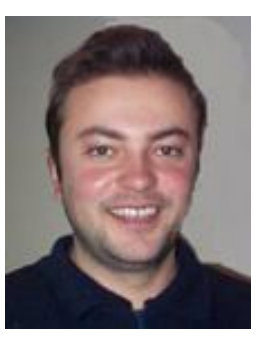

Andrej Stefanov (M'01) received the B.S. degree in electrical engineering from Cyril and Methodius University, Skopje, Macedonia, in 1996. He received the M.S. and Ph.D. degrees in electrical engineering from Arizona State University, Tempe, AZ, in 1998 and 2001, respectively. During the summer 2000, he was with the Advanced Development Group, Hughes Network Systems, Germantown, MD. He was an assistant professor at the Electrical and Computer Engineering Department of the Polytechnic Institute of NYU (Brooklyn Poly) between 2001 and 2008. He was a visiting scientist at Northeastern University between 2009 and 2011. He joined the Faculty at IBU Skopje in 2011. Dr. Stefanov is the recipient of the IEEE Benelux Joint Chapter Best Paper Award at IEEE VTCFall 1999, Amsterdam, The Netherlands. He served as an Editor for the IEEE Transactions on Wireless Communications, 2006-2008. His research interests are in communication theory, wireless and mobile communications, wireless networks, and channel coding.

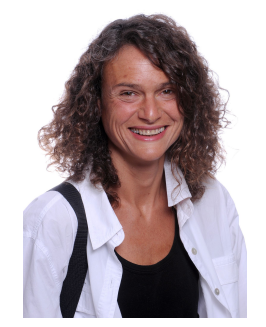

Milica Stojanovic (Sm08,F10) graduated from the University of Belgrade, Serbia, in 1988, and received the M.S. and Ph.D. degrees in electrical engineering from Northeastern University, Boston, MA, in 1991 and 1993. After a number of years with the Massachusetts Institute of Technology, where she was a Principal Scientist, she joined the faculty of Electrical and Computer Engineering Department at Northeastern University in 2008. She is also a Guest Investigator at the Woods Hole Oceanographic Institution, and a Visiting Scientist at MIT. Her research interests include digital communications theory, statistical signal processing and wireless networks, and their applications to underwater acoustic communication systems. Milica is an Associate Editor for the IEEE Journal of Oceanic Engineering and the IEEE Transactions on Signal processing. 